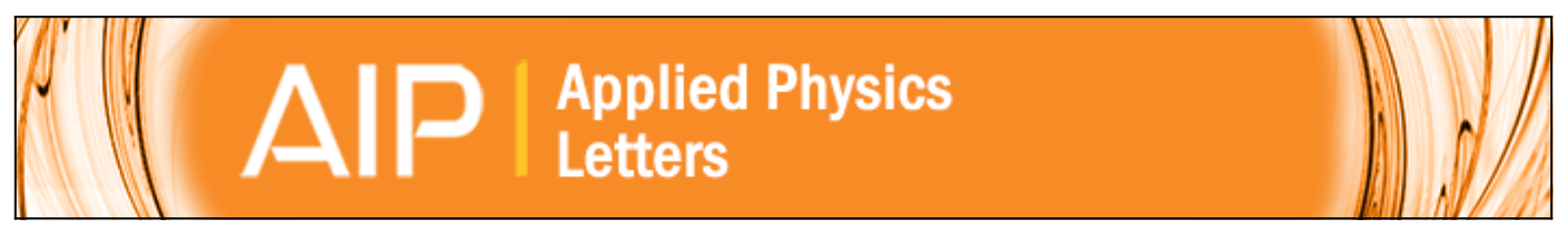

\title{
Excellent magnetocaloric properties of melt-extracted Gd-based amorphous microwires
}

N. S. Bingham, H. Wang, F. Qin, H. X. Peng, J. F. Sun, V. Franco, H. Srikanth, and M. H. Phan

Citation: Applied Physics Letters 101, 102407 (2012); doi: 10.1063/1.4751038

View online: http://dx.doi.org/10.1063/1.4751038

View Table of Contents: http://scitation.aip.org/content/aip/journal/apl/101/10?ver=pdfcov

Published by the AIP Publishing

\section{Articles you may be interested in}

Table-like magnetocaloric effect in Gd56Ni15Al27Zr2 alloy and its field independence feature

J. Appl. Phys. 118, 123903 (2015); 10.1063/1.4931765

Impact of structural disorder on the magnetic ordering and magnetocaloric response of amorphous Gd-based microwires

J. Appl. Phys. 115, 17 A318 (2014); 10.1063/1.4864143

Magnetic and reversible magnetocaloric properties of ( Gd $1-x$ Dy x ) 4 Co 3 ferrimagnets

J. Appl. Phys. 105, 053902 (2009); 10.1063/1.3075627

Large magnetic entropy changes in $\mathrm{Gd}-\mathrm{Co}$ amorphous ribbons

J. Appl. Phys. 105, 013912 (2009); 10.1063/1.3040009

Magnetocaloric effect in Gd-based bulk metallic glasses

Appl. Phys. Lett. 89, 081914 (2006); 10.1063/1.2338770

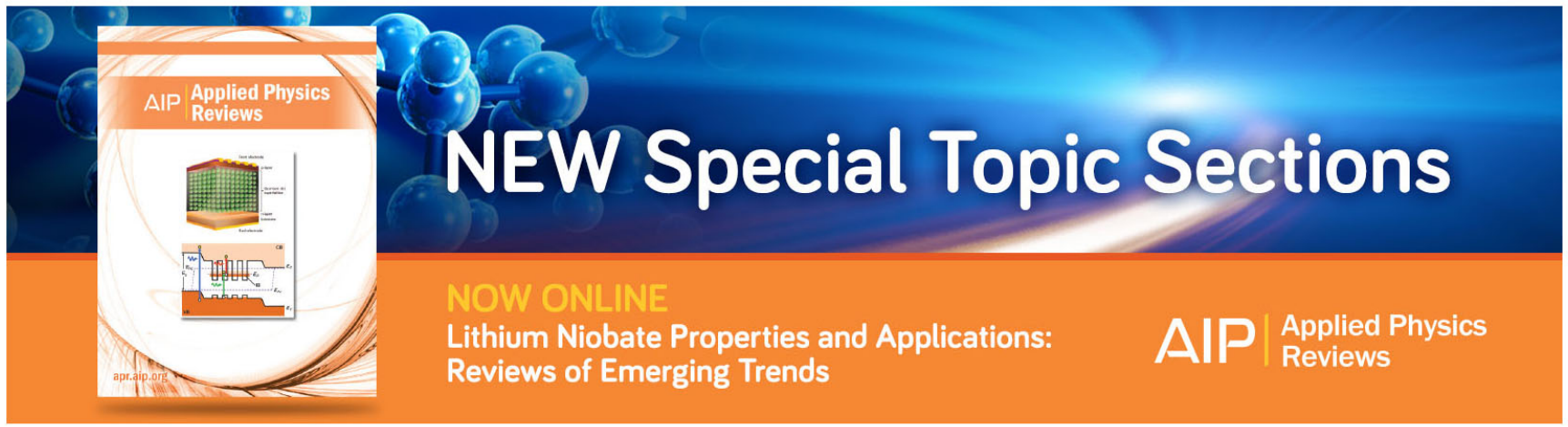




\title{
Excellent magnetocaloric properties of melt-extracted Gd-based amorphous microwires
}

\author{
N. S. Bingham, ${ }^{1, a)}$ H. Wang, ${ }^{2, a)}$ F. Qin, ${ }^{3, b)}$ H. X. Peng, ${ }^{3}$ J. F. Sun, ${ }^{2}$ V. Franco, ${ }^{4}$ H. Srikanth, ${ }^{1, b)}$ \\ and M. H. Phan ${ }^{1, b)}$ \\ ${ }^{1}$ Department of Physics, University of South Florida, Tampa, Florida 33620, USA \\ ${ }^{2}$ School of Materials Science and Engineering, Harbin Institute of Technology, Harbin 150001, China \\ ${ }^{3}$ Advanced Composite Center for Innovation and Science, Department of Aerospace Engineering, \\ University of Bristol, University Walk, Bristol BS8 1TR, United Kingdom \\ ${ }^{4}$ Dpto. Fisica de la Materia Condensada, ICMSE-CSIC, Universidad de Sevilla, P.O. Box 1065, \\ 41080 Sevilla, Spain
}

(Received 4 July 2012; accepted 23 August 2012; published online 6 September 2012)

\begin{abstract}
We report upon the excellent magnetocaloric properties of $\mathrm{Gd}_{53} \mathrm{Al}_{24} \mathrm{Co}_{20} \mathrm{Zr}_{3}$ amorphous microwires. In addition to obtaining the large magnetic entropy change $\left(-\Delta S_{M} \sim 10.3 \mathrm{~J} / \mathrm{kg} \mathrm{K}\right.$ at $T_{C} \sim 95 \mathrm{~K}$ ), an extremely large value of refrigerant capacity $(\mathrm{RC} \sim 733.4 \mathrm{~J} / \mathrm{kg}$ ) has been achieved for a field change of $5 \mathrm{~T}$ in an array of forty microwires arranged in parallel. This value of $\mathrm{RC}$ is about $79 \%$ and $103 \%$ larger than those of $\mathrm{Gd}(\sim 410 \mathrm{~J} / \mathrm{kg})$ and $\mathrm{Gd}_{5} \mathrm{Si}_{2} \mathrm{Ge}_{1.9} \mathrm{Fe}_{0.1}(\sim 360 \mathrm{~J} / \mathrm{kg})$ regardless of their magnetic ordering temperatures. The design and fabrication of a magnetic bed made of these parallel-arranged microwires would thus be a very promising approach for active magnetic refrigeration for nitrogen liquefaction. Since these microwires can easily be assembled as laminate structures, they have potential applications as a cooling device for micro electro mechanical systems and nano electro mechanical systems. (c) 2012 American Institute of Physics. [http://dx.doi.org/10.1063/1.4751038]
\end{abstract}

Magnetic refrigeration (MR) based on the magnetocaloric effect (MCE) is an energy-efficient and environmentally friendly cooling technology. ${ }^{1-3}$ Using a magnetic substance as a refrigerant material and the principle of "magnetizing" and "demagnetizing", this technology provides a higher cooling efficiency and compactness over conventional gas compression techniques. A main goal for making this technology possible is to seek materials that are cost-effective and exhibit large magnetic entropy change $\left(\Delta S_{\mathrm{M}}\right)$ over a wide temperature range, namely a large refrigerant capacity $(\mathrm{RC}) .^{4-13}$ The $\mathrm{RC}$ is a measure of the amount of heat transfer between the cold and hot reservoirs in an ideal refrigeration cycle, which depends not only on the magnitude of $\Delta \mathrm{S}_{\mathrm{M}}$ but also on its temperature dependence (e.g., the full width at half maximum of the $\Delta S_{M}(T)$ peak). ${ }^{4,14}$ The existing magnetocaloric materials are far from ideal due to the limitations of their intrinsic structure and properties. Specifically, materials with a first-order magnetic transition (FOMT), such as Gd-Si-Ge, ${ }^{1} \mathrm{Mn}-\mathrm{Fe}-\mathrm{P}-\mathrm{As},{ }^{15} \mathrm{Mn}-$ As-Sb, ${ }^{16}$ and Ni-Mn-Ga ${ }^{17}$ alloys, possess a larger $\Delta \mathrm{S}_{\mathrm{M}}$, but the effect is restricted to a narrow temperature range resulting in a smaller RC. Materials displaying a second-order magnetic transition (SOMT) are often found to exhibit a smaller MCE but with a distribution over a broader temperature range, thus resulting in a larger RC. . $3,4,9,18^{\text {In addition, }}$ hysteretic losses associated with the FOMT are usually very large and therefore detrimental to the $\mathrm{RC}$, whereas these effects are very small or negligible in the case of the SOMT materials. ${ }^{4,18}$ In this context, it would be desirable to engi-

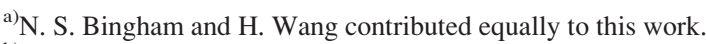

b) Electronic addresses: faxiang.qin@bris.ac.uk, sharihar@usf.edu, and phanm@usf.edu.
}

neer peculiar SOMT materials ${ }^{1,3-5,7,9}$ or composites ${ }^{6,9-13}$ that undergo multiple SOMTs to exhibit large RC for active magnetic refrigeration (AMR) applications.

From a cooling application perspective, it is noted that a magnetic refrigerator acquires a higher amount of heat absorption/extraction per volume than in a conventional gas-based cooling system. ${ }^{1,19}$ Since a magnetic material is the working body of a MR system, it is required to have a large heat transfer area to provide high heat exchange efficiency. ${ }^{19}$ In this context, the exploration of the MCE in magnetic materials in the form of micro/nanowires is of practical importance. ${ }^{20-22}$ The use of these wires with increased surface areas will not only enhance heat transfer processes but also promote chemical reactions of solid refrigerant with liquid coolant used to transfer heat inside the system. ${ }^{19}$ However, the MCEs achieved in the existing micro/nanowires ${ }^{20-22}$ were relatively small (e.g., $-\Delta S_{M} \sim 0.7 \mathrm{~J} / \mathrm{kg} \mathrm{K}$ at $3 \mathrm{~T}$ for $\mathrm{Ni}_{50.95} \mathrm{Mn}_{25.45} \mathrm{Ga}_{23.6}$ microwires ${ }^{22}$ ), making them unsuitable for AMR applications.

In this letter, the excellent magnetocaloric properties of Gd-based amorphous microwires, which were fabricated by a modified precision melt-extraction method, are reported. The large values of MCE $\left(-\Delta S_{M} \sim 10.3 \mathrm{~J} / \mathrm{kg} \mathrm{K}\right.$ at $\left.T_{C} \sim 95 \mathrm{~K}\right)$ and $\mathrm{RC}(\sim 733.4 \mathrm{~J} / \mathrm{kg})$ are achieved for a field change of $5 \mathrm{~T}$ in an array of forty parallelly arranged microwires. This value of RC is about $79 \%$ larger than that of $\mathrm{Gd}(\sim 410 \mathrm{~J} / \mathrm{kg})$ for the same field change regardless of their ordering temperature. In addition, negligible thermal and field hysteresis losses are observed in these wires, due to the nature of SOMT. These excellent magnetocaloric properties make this system one of the best candidate materials for AMR in the liquid nitrogen temperature range.

Amorphous wires of a nominal composition $\mathrm{Gd}_{53} \mathrm{Al}_{24}$ $\mathrm{Co}_{20} \mathrm{Zr}_{3}$ were fabricated using a home-built melt-extraction 
facility. ${ }^{23}$ The composition of the wire was chosen because its bulk glass counterpart was reported to show excellent magnetocaloric properties. ${ }^{5}$ A fabrication process of the wires can be described as follows: The ingot with a diameter of $8 \mathrm{~mm}$ was prepared from raw materials $\mathrm{Gd}, \mathrm{Al}, \mathrm{Co}$, and $\mathrm{Zr}$ crystals in argon atmosphere by arc melting. Melt extraction process was performed using a copper wheel with a diameter of $160 \mathrm{~mm}$ and $60^{\circ} \mathrm{knife}$ edge, the linear velocity of the wheel rim was fixed at $30 \mathrm{~m} / \mathrm{min}$ and a feed rate of the molten was kept at $90 \mu \mathrm{m} / \mathrm{s}$. The amorphous nature of the wires was confirmed by X-ray diffraction (XRD). The morphology of the wires was observed by a field emission scanning electron microscope (SEM S-4700) at $15 \mathrm{kV}$. Thermal analysis was carried out in a Perkin-Elmer differential scanning calorimeter (DSC) at a heating rate of $20 \mathrm{~K} / \mathrm{min}$. Magnetic and magnetocaloric measurements were conducted using a commercial physical property measurement system (PPMS) from quantum design with a temperature range of 5-300 K and applied fields up to $7 \mathrm{~T}$.

Figure 1 shows DSC (main panel) and a side-view image (inset) of the fabricated wires. The DSC profile clearly reveals the glassy nature of the wires. The endothermic reaction displayed in the graph indicates the glass transition. The crystallization is reflected by the presence of a sharp exothermic peak. The glass transition temperature $T_{g}$ and onset crystallization temperature $T_{x}$ are determined to be about $602 \mathrm{~K}$ and $651 \mathrm{~K}$, respectively. The resulting width of the supercooled region $\Delta T_{x}=T_{x}-T_{g}$ yields a value of $49 \mathrm{~K}$, which is similar to that reported by Luo et al. ${ }^{5}$ The large value of $\Delta T_{x}$ obtained, coupled with the observed distinctive glass transition and sharp crystallization, suggests the excellent glass forming ability of the fabricated wires. As can be seen in the inset of Fig. 1, wires of an average diameter of $50 \mu \mathrm{m}$ were fabricated.

On investigating the magnetocaloric properties of the present wires, we recall that there is a noticeable influence of the demagnetizing field (e.g., the shape of a sample) on the determination of the MCE from isothermal M-H curves ${ }^{24}$ and that the shape anisotropy plays an important role in determining the MCE in an array of parallel-arranged nanowires. ${ }^{21}$ Therefore, in the present work, we have performed a comparative study of the magnetic and magnetocaloric properties of the wire-shaped sample (the average length is $5 \mathrm{~mm}$ ) and the bulk rectangular-shaped sample (the length, width, and thickness are $2.5 \mathrm{~mm}, 1.78 \mathrm{~mm}$, and $0.76 \mathrm{~mm}$, respectively). While

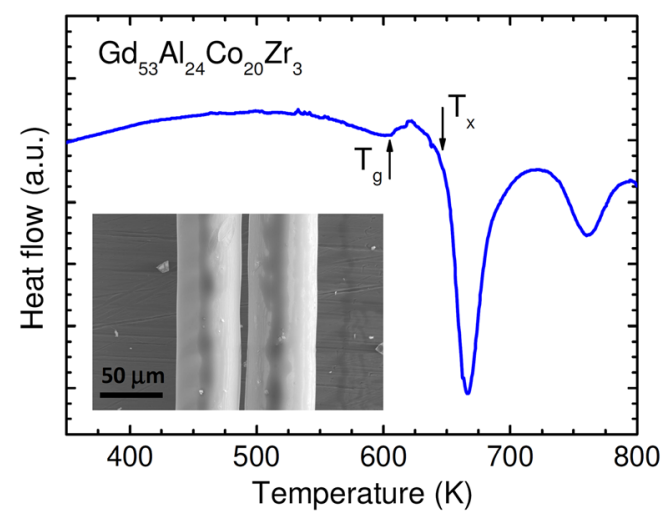

FIG. 1. DSC curve of a $\mathrm{Gd}_{53} \mathrm{Al}_{24} \mathrm{Co}_{20} \mathrm{Zr}_{3}$ amorphous microwire. The inset shows a side-view image of the microwires.
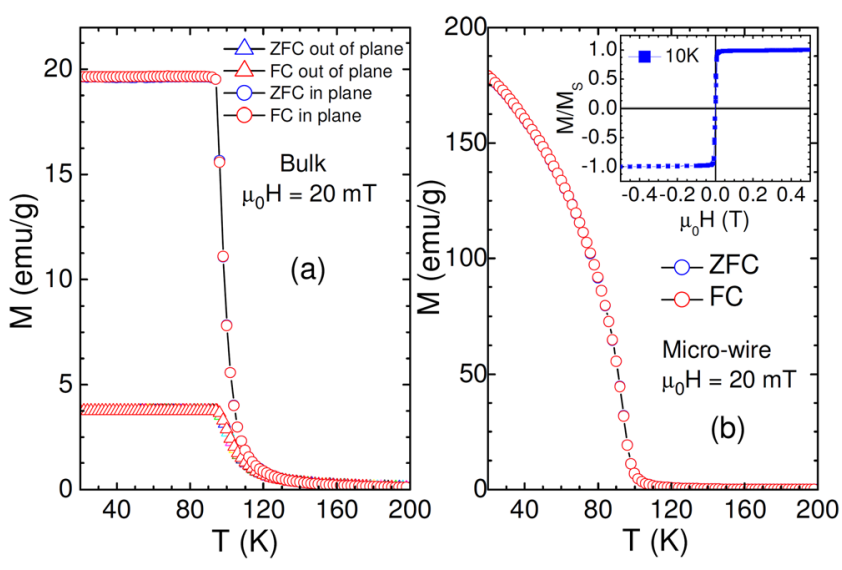

FIG. 2. Temperature dependence of ZFC and FC magnetization taken at a field of $20 \mathrm{mT}$ (a) for the bulk sample (for both "in-plane" and "out-ofplane" cases) and (b) for the microwires sample. The inset shows the magnetic hysteresis loop (M-H) of the microwires sample.

the magnetic field was applied along the microwires during magnetic measurements, it was applied in-plane and out-ofplane to the rectangular-shaped sample. Figure 2 shows the temperature dependence of zero-field-cooled (ZFC) and fieldcooled (FC) magnetization taken at a field of $20 \mathrm{mT}$ for the bulk sample (Fig. 2(a), in-plane and out-of-plane) and for the microwires sample (Fig. 2(b)). It can be observed that the samples undergo a paramagnetic to ferromagnetic (PM-FM) transition. The Curie temperature $T_{C}$, which is defined by the minimum in $\mathrm{dM} / \mathrm{dT}$, is determined to be about $95 \mathrm{~K}$ for the samples studied. It is also noted that the magnetization is much larger and the PM-FM transition is sharper for the "inplane" case than for the "out-of-plane" case due to a lower demagnetizing factor. The microwires sample exhibits a broad PM-FM transition, with a very large value of magnetization observed at low temperatures. As one can see in the inset of Fig. 2(b), the $M-H$ loop taken at $10 \mathrm{~K}$ shows a soft ferromagnetic characteristic $\left(H_{C} \sim 0.8 \mathrm{mT}\right)$ for the microwires, which is desirable for AMR. The square shape of the $M-H$ loop consistently indicates that the easy direction of the magnetization is parallel to the microwire axis.

In order to evaluate the MCE in the present samples, the isothermal magnetization curves of the samples were measured with a field step of $0.05 \mathrm{mT}$ in a range of $0-3 \mathrm{~T}$ and a temperature interval of $3 \mathrm{~K}$ in a range of temperatures around $T_{C}$. The results are shown in Figs. 3(a)-3(c) for all three cases. It is worth noting that unlike the bulk sample (both in-plane and out-of-plane), the magnetization easily reaches its saturation state at low magnetic fields for the case of the microwires sample, due to the shape anisotropy of the microwires. Interestingly, large variations in the magnetization around the $T_{C}$ occur at low magnetic fields $(<2 \mathrm{~T})$, indicating a large low-magnetic field induced magnetic entropy change in the microwires sample. This feature is desirable for domestic applications of magnetic refrigerators, where, instead of using expensive superconducting magnets, more economical permanent magnets can be used as a magnetic field source. ${ }^{2}$

To assess the nature of the magnetic phase transitions in the present samples, Arrott plots have been constructed based on the $M-H$ data (Figs. 3(d)-3(f)). According to the Banerjee criterion, ${ }^{25}$ the sign of the slope of $H / M$ vs $M^{2}$ is determined by the nature of a PM to FM transition, with a negative slope 

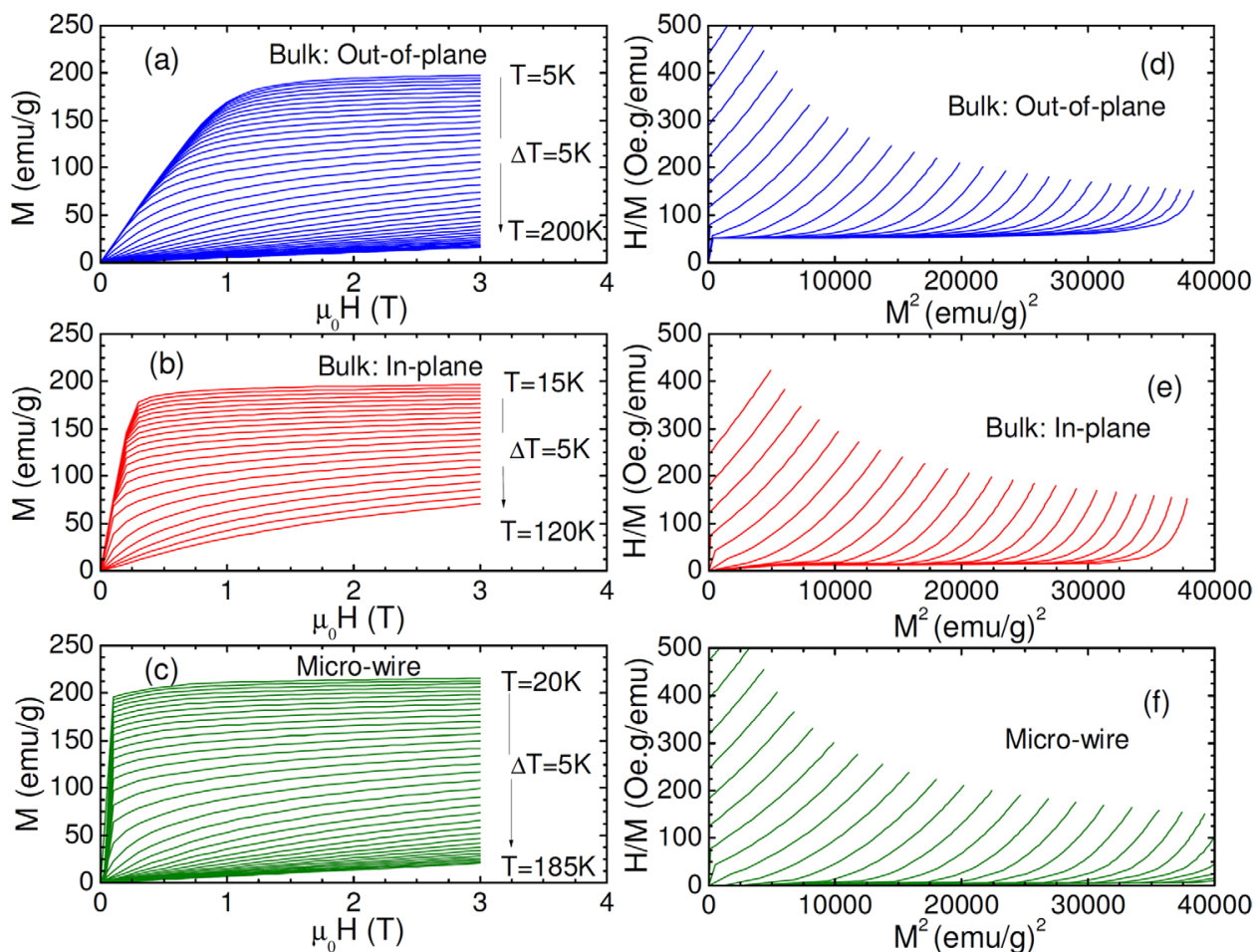

FIG. 3. Isothermal magnetization curves taken at different fixed temperatures between $5 \mathrm{~K}$ and $300 \mathrm{~K}$ ((a) and (b)) for the bulk sample (the "in-plane" and "out-of-plane" cases, respectively) and (c) for the microwires sample. The $H / M$ vs. $M^{2}$ plots for representative temperatures around the $\mathrm{T}_{\mathrm{C}}$ for the (d) "inplane," (e) "out-of-plane," and (f) "microwire" cases, respectively. corresponding to a first order transition and a positive slope corresponding to a second order transition, which is also supported by the lack of thermal hysteresis. By this criterion, all the present samples clearly undergo a second-order magnetic transition, which is desirable for AMR since first-order transitions are accompanied by large hysteretic losses. ${ }^{18}$

From the $M-H$ isotherms shown in Figs. 3(a)-3(c), the magnetic entropy change $\left(\Delta S_{M}\right)$ of the samples has been calculated using the Maxwell relation, ${ }^{18}$

$$
\Delta S_{M}=\mu_{0} \int_{0}^{H_{\max }}\left(\frac{\partial M}{\partial T}\right)_{H} d H,
$$

where $M$ is the magnetization, $H$ is the magnetic field, and $T$ is the temperature. Figures 4(a) and 4(b) show the temperature dependence of $-\Delta S_{M}$ at different magnetic field changes up to $3 \mathrm{~T}$ for the bulk sample (the "out-of-plane" case) and for the microwires sample, respectively. As expected, both samples exhibit large magnetic entropy changes around the $T_{C}$. From the data shown in Figs. 4(a) and 4(b), the magnetic field dependence of the maximum magnetic entropy change $\left(\Delta S_{M}^{\max }\right)$ has been deduced and is plotted in Fig. 4(c). It is interesting to note in this figure that the value of $\Delta S_{M}^{\max }$ is largest for the case of the microwires sample and is smallest for the "out-of-plane" case of the bulk sample. Another remarkable feature is that the $-\Delta S_{M}$ vs. $T$ curves are broader in the case of the microwires sample. This thus leads to an expectation that a much larger $\mathrm{RC}$ would be achieved in this sample. To confirm this, the RC of the samples has been calculated from the relation

$$
R C=-\int_{T_{1}}^{T_{2}} \Delta S_{M}(T) d T,
$$

where $T_{1}$ and $T_{2}$ are the temperatures of the cold end and the hot end of an ideal thermodynamic cycle, respectively. ${ }^{14}$ This is plotted as a function of magnetic field and the result is displayed in Fig. 4(d) for all three cases. The largest value of $\mathrm{RC}$ is achieved in the microwires sample. For $\mu_{0} \Delta H=2 \mathrm{~T}$, the RC calculated for the microwires sample is $276.2 \mathrm{~J} / \mathrm{kg}$, while it is $224.3 \mathrm{~J} / \mathrm{kg}$ and $166.3 \mathrm{~J} / \mathrm{kg}$ for the "in-plane" and "out-of-plane" cases of the bulk sample, respectively. To put our results in the context of previous studies, we summarize in Table I the $-\Delta S_{M}$ and RC values of the present samples and other magnetocaloric candidate materials for AMR in the temperature range of $10-100 \mathrm{~K}$. We have used the method $^{26}$ based on the power law relationship between RC and magnetic field to evaluate the $\mathrm{RC}$ of the present samples for $\mu_{0} \Delta H=5 \mathrm{~T}$. It can be seen in Table I that among the compared magnetocaloric materials, ${ }^{3-5,27,28}$ the microwires sample shows the largest RC. For $\mu_{0} \Delta H=5 \mathrm{~T}$, the value of $\mathrm{RC}(\sim 733.4 \mathrm{~J} / \mathrm{kg})$ achieved for the microwires sample is about $79 \%$ and $103 \%$ larger than those of $\mathrm{Gd}(\sim 410 \mathrm{~J} / \mathrm{kg})^{3}$ and $\mathrm{Gd}_{5} \mathrm{Si}_{2} \mathrm{Ge}_{1.9} \mathrm{Fe}_{0.1}(\sim 360 \mathrm{~J} / \mathrm{kg})^{4}$ regardless of their ordering temperature. We recall that the microwires also possess zero thermal and nearly zero field hysteresis losses, due to the nature of SOMT. These excellent magnetocaloric properties make it one of the best candidate materials for AMR in the liquid nitrogen temperature range.

As reported previously in Refs. 24 and 29, the increase of the demagnetizing factor $(N)$ caused a significant decrease in $\Delta S_{M}$ for temperatures below $T_{C}$, while the shape of the $\Delta S_{M}$ vs. $T$ curves remained unchanged for temperatures above $T_{C}$. In the present study, the values of $N$ are determined to be $\sim 0$ for the "microwire" and "in-plane" cases, while it is 1 for the "out-of-plane" case. We have observed a significant effect of the sample shape on the $\Delta S_{M}$ and RC in $\mathrm{Gd}_{53} \mathrm{Al}_{24} \mathrm{Co}_{20} \mathrm{Zr}_{3}$ and that the microwires sample shows the largest values of $\Delta S_{M}$ and RC resulting from the $N \sim 0$ and the high shape anisotropy of the wire. Since it is an array of magnetic microwires, dipolar magnetic interactions among microwires in the array may also play a role in enhancing the $\Delta S_{M}$ and $\mathrm{RC}$ of the $\mathrm{Gd}_{53} \mathrm{Al}_{24} \mathrm{Co}_{20} \mathrm{Zr}_{3}$ microwires. However, 

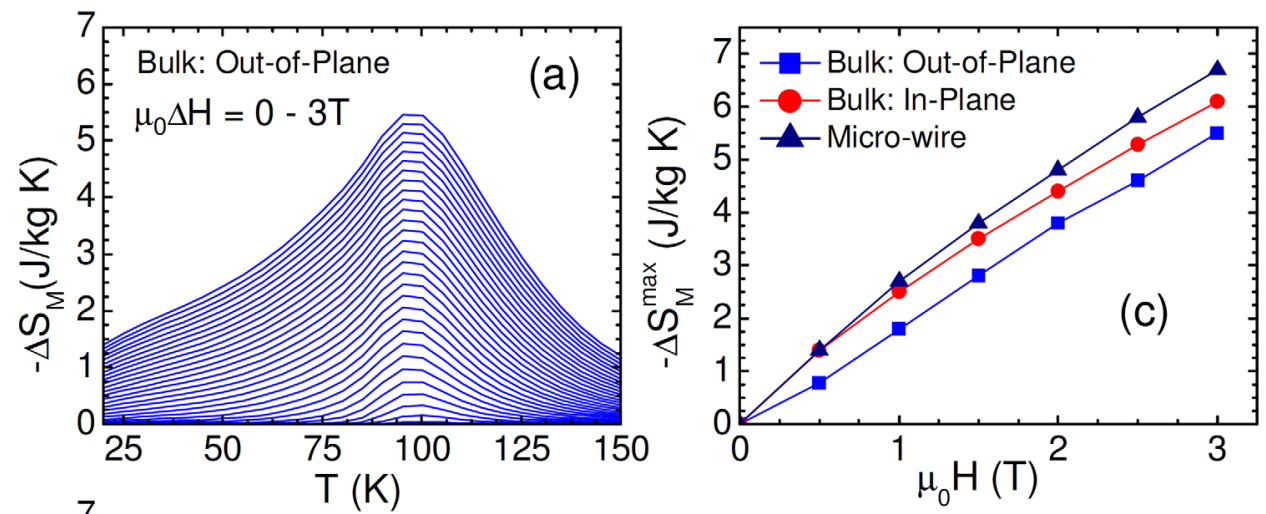
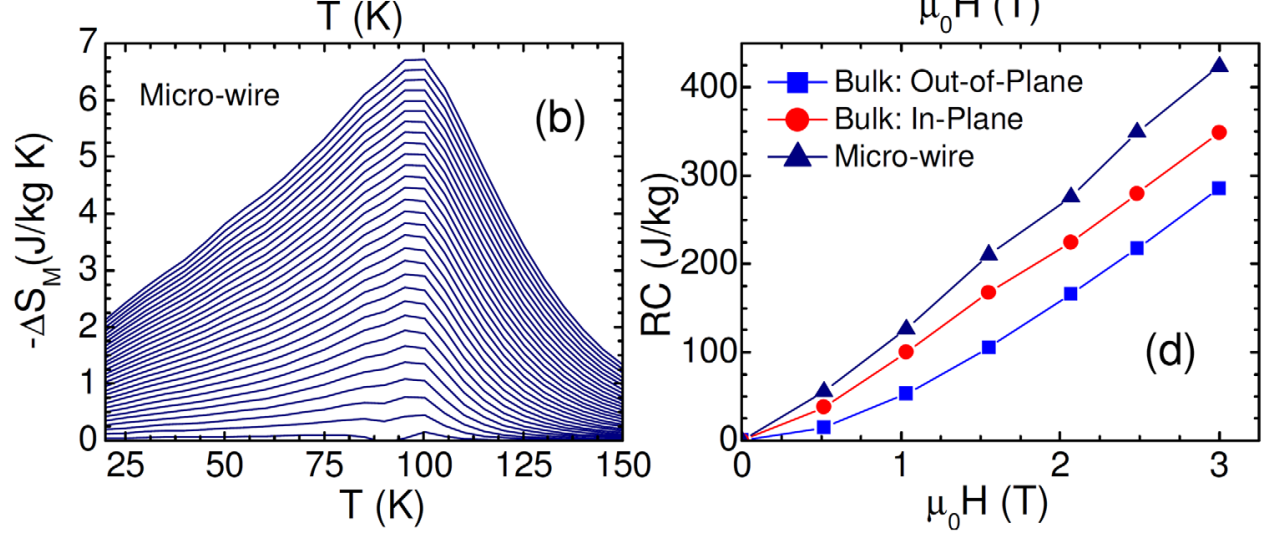

FIG. 4. Temperature dependence of magnetic entropy change $\left(-\Delta S_{M}\right)$ for different field changes up to $3 \mathrm{~T}$ (a) for the bulk sample (the "out-of-plane" case) and (b) for the microwires sample. Magnetic field dependences of maximum magnetic entropy change $\left(\Delta \mathrm{S}_{\mathrm{M}}^{\max }\right)$ (Fig. 4(c)) and RC (Fig. 4(d)) for all the "in-plane," "out-of-plane," and "microwire" cases. further study is needed to clarify this. It has also been noted that due to the poor geometry and associated stress and chemical inhomogeneity, the demagnetizing field is reinforced and non-uniformly distributed with the addition of induced local demagnetizing field in the wire, which, in effect, degrades the magnetic properties including MCE. ${ }^{30}$ The magnified SEM images of the $\mathrm{Gd}_{53} \mathrm{Al}_{24} \mathrm{Co}_{20} \mathrm{Zr}_{3}$ microwires reveal the presence of a few bumps and high surface roughness. Therefore, there remains large room to enhance the quality of the present wires via optimization of fabrication process so as to further improve the MCE performance.

In summary, we have fabricated the amorphous microwires of $\mathrm{Gd}_{53} \mathrm{Al}_{24} \mathrm{Co}_{20} \mathrm{Zr}_{3}$ using a modified precision melt-extraction method. These microwires show excellent magnetocaloric properties, which are certainly open to further optimization via facile processing manipulation. We propose that the design and fabrication of a magnetic bed made of these multiple single parallel-arranged microwires would be a very promising approach for active magnetic refrigeration for nitrogen liquefaction.

The work at the University of South Florida was supported by the Department of Army through Grant No. W911NF-08-1-0276. M.H.P. acknowledges the support from the Florida Cluster for Advanced Smart Sensor Technologies (FCASST). F.X.Q. would like to acknowledge the financial support from EPSRC Institutional Sponsorship Fund. V.F. acknowledges the financial support of the Spanish Ministry of Science and Innovation and EU FEDER (Project MAT 2010-20537), the PAI of the Regional Government of Andalucía (Project P10-FQM-6462), and the United States Office of Naval Research (Project N00014-11-1-0311).

TABLE I. Maximum entropy change, $\left|\Delta S_{M}^{\max }\right|$, peak temperature, $T_{\mathrm{p}}$, and $\mathrm{RC}$, for the present samples and other magnetocaloric candidate materials (Bulk Glasses) in the temperature range of $10-100 \mathrm{~K}$. Gd and $\mathrm{Gd}_{5} \mathrm{Si}_{2} \mathrm{Ge}_{1.9} \mathrm{Fe}_{0.1}$ are also included for reference.

\begin{tabular}{|c|c|c|c|c|c|}
\hline Material & $T_{\mathrm{p}}(\mathrm{K})$ & $\mu_{0} \Delta \mathrm{H}(\mathrm{T})$ & $\left|\Delta S_{M}^{\max }\right|(\mathrm{J} / \mathrm{kg} \mathrm{K})$ & $\mathrm{RC}(\mathrm{J} / \mathrm{kg})$ & Reference \\
\hline $\mathrm{Gd}_{53} \mathrm{Al}_{24} \mathrm{Co}_{20} \mathrm{Zr}_{3}$ (Micro-wire) & 96 & 5 & 10.3 & 733.4 & Present \\
\hline $\mathrm{Gd}_{53} \mathrm{Al}_{24} \mathrm{Co}_{20} \mathrm{Zr}_{3}$ (Bulk, in-plane) & 95 & 5 & 9.6 & 609.2 & Present \\
\hline $\mathrm{Gd}_{53} \mathrm{Al}_{24} \mathrm{Co}_{20} \mathrm{Zr}_{3}$ (Bulk, out-of-plane) & 95 & 5 & 9.1 & 546.4 & Present \\
\hline $\mathrm{Gd}_{53} \mathrm{Al}_{24} \mathrm{Co}_{20} \mathrm{Zr}_{3}($ Bulk $)$ & 93 & 5 & 9.4 & 590 & 5 \\
\hline $\mathrm{Gd}_{33} \mathrm{Al}_{25} \mathrm{Co}_{20} \mathrm{Er}_{22}$ (Bulk) & 52 & 5 & 9.47 & 574 & 5 \\
\hline $\mathrm{Gd}_{51} \mathrm{Al}_{24} \mathrm{Co}_{20} \mathrm{Nb}_{1} \mathrm{Cr}_{4}$ (Bulk) & 100 & 5 & 9.48 & 611 & 27 \\
\hline $\mathrm{Gd}_{48} \mathrm{Al}_{25} \mathrm{Co}_{20} \mathrm{Zr}_{3} \mathrm{Er}_{4}$ (Bulk) & 84 & 5 & 9.41 & 647 & 27 \\
\hline $\mathrm{Gd}_{51} \mathrm{Al}_{24} \mathrm{Co}_{20} \mathrm{Zr}_{4} \mathrm{Nb}_{1}$ (Bulk) & 91 & 5 & 9.23 & 651 & 27 \\
\hline $\mathrm{Dy}_{50} \mathrm{Gd}_{7} \mathrm{Al}_{23} \mathrm{Co}_{20}$ (Bulk) & 26 & 5 & 9.77 & 290 & 27 \\
\hline $\mathrm{Gd}_{60} \mathrm{Co}_{26} \mathrm{Al}_{14}($ Bulk $)$ & 79 & 5 & 10.1 & 557 & 27 \\
\hline $\mathrm{Gd}_{55} \mathrm{Co}_{20} \mathrm{Al}_{25}$ (Bulk) & 103 & 5 & 8.8 & 541 & 28 \\
\hline $\mathrm{Gd}_{55} \mathrm{Co}_{25} \mathrm{Ni}_{20}$ (Bulk) & 78 & 5 & 8.0 & 640 & 28 \\
\hline Gd (bulk) ${ }^{\mathrm{a}}$ & 294 & 5 & 10.2 & 410 & 3 \\
\hline $\mathrm{Gd}_{5} \mathrm{Si}_{2} \mathrm{Ge}_{1.9} \mathrm{Fe}_{0.1}(\mathrm{Bulk})^{\mathrm{a}}$ & 305 & 5 & 7.0 & 360 & 4 \\
\hline
\end{tabular}

${ }^{\mathrm{a}}$ Crystalline structure. 
${ }^{1}$ V. K. Pecharsky, K. A. Gschneidner, and A. O. Tsokol, Rep. Prog. Phys. 68, 1479 (2005).

${ }^{2}$ E. Bruck, J. Phys. D: Appl. Phys. 38, R381 (2005).

${ }^{3}$ M. H. Phan and S. C. Yu, J. Magn. Magn. Mater. 308, 325 (2007).

${ }^{4}$ V. Provenzano, A. J. Shapiro, and R. D. Shull, Nature (London) 429, 853 (2004).

${ }^{5}$ Q. Luo, D. Q. Zhao, M. X. Pan, and W. H. Wang, Appl. Phys. Lett. 89, 081914 (2006).

${ }^{6}$ S. Gorsse, B. Chevalier, and G. Orveillon, Appl. Phys. Lett. 92, 122501 (2008).

${ }^{7}$ P. Kumar, N. K. Singh, K. G. Suresh, and A. K. Nigam, Phys. Rev. B 77, 184411 (2008).

${ }^{8}$ M. H. Phan, S. Chandra, N. S. Bingham, H. Srikanth, C. L. Zhang, S. W. Cheong, T. D. Hoang, and H. D. Chinh, Appl. Phys. Lett. 97, 242506 (2010).

${ }^{9}$ V. Franco and A. Conde, Scripta Mater. 67, 594 (2012).

${ }^{10}$ R. Caballero-Flores, V. Franco, A. Conde, K. E. Knipling, and M. A. Willard, Appl. Phys. Lett. 98, 102505 (2011).

${ }^{11}$ A. Chaturvedi, S. Stefanoski, M. H. Phan, G. S. Nolas, and H. Srikanth, Appl. Phys. Lett. 99, 162513 (2011).

${ }^{12}$ P. Alvarez, J. L. Sánchez Llamazares, P. Gorria, and J. A. Blanco, Appl. Phys. Lett. 99, 232501 (2011).

${ }^{13}$ S. C. Paticopoulos, R. Caballero-Flores, V. Franco, J. S. Blázquez, A. Conde, K. E. Knipling, and M. A. Willard, Solid State Comm. 152, 1590 (2012).

${ }^{14}$ V. K. Pecharsky and K. A. Gschneidner, Jr., J. Appl. Phys. 90, 4614 (2001).

${ }^{15}$ O. Tegus, E. Bruck, L. Zhang, W. Dagula, K. H. J. Buschow, and F. R. De Boer, Physica B 319, 174 (2002).
${ }^{16}$ H. Wada and Y. Tanabe, Appl. Phys. Lett. 79, 3302 (2001).

${ }^{17}$ F. X. Hu, B. G. Shen, and J. R. Sun, Appl. Phys. Lett. 76, 3460 (2000).

${ }^{18}$ N. S. Bingham, M. H. Phan, H. Srikanth, M. A. Torija, and C. Leighton, J. Appl. Phys. 106, 023909 (2009).

${ }^{19}$ C. R. H. Bah, D. Velázquez, K. K. Nielsen, K. Engelbrecht, K. B. Andersen, R. Bulatova, and N. Pryds, Appl. Phys. Lett. 100, 121905 (2012).

${ }^{20}$ M. I. Ilyn, V. Zhukova, J. D. Santos, M. L. Sánchez, V. M. Prida, B. Hernando, V. Larin, J. González, A. M. Tishin, and A. Zhukov, Phys. Status Solidi A 205, 1378 (2008).

${ }^{21}$ V. Franco, K. R. Pirota, V. M. Prida, A. M. J. C. Neto, A. Conde, M. Knobel, B. Hernando, and M. Vazquez, Phys. Rev. B 77, 104434 (2008).

${ }^{22}$ R. Varga, T. Ryba, Z. Vargova, K. Saksl, V. Zhukova, and A. Zhukov, Scr. Mater. 65, 703 (2011).

${ }^{23}$ H. Wang, D. W. Xing, X. D. Wang, and J. F. Sun, Metall. Mater. Trans. A 42(4), 1103 (2010).

${ }^{24}$ R. Caballero-Flores, V. Franco, A. Conde, and L. F. Kiss, J. Appl. Phys. 105, 07A919 (2009).

${ }^{25}$ S. K. Banerjee, Phys. Lett. 12, 16 (1964).

${ }^{26}$ V. Franco and A. Conde, Int. J. Refrig. 33, 465 (2010).

${ }^{27}$ W. H. Wang, Adv. Mater. 21, 4524 (2009).

${ }^{28}$ J. Du, Q. Zheng, Y. B. Li, Q. Zhang, D. Li, and Z. D. Zhang, J. Appl. Phys. 103, 023918 (2008).

${ }^{29}$ C. R. H. Bahl and K. K. Neilsen, J. Appl. Phys. 105, 013916 (2009).

${ }^{30}$ A. Zhukov and V. Zhukova, Magnetic Properties and Applications of Ferromagnetic Mircowires with Amorphous and Nanocrystalline Structure (Nova Science, New York, 2009). 